\title{
Charged-current quasi-elastic scattering at MINERvA
}

Cheryl Patrick*†

University College London (formerly Northwestern University)

E-mail: c.patrick@ucl.ac.uk

Charged-current quasi-elastic scattering is a particularly interesting interaction to understand. Not only is it a key signal process for oscillation experiments, but its relatively clean signature makes it an ideal channel to study nuclear effects, such as multi-nucleon effects, in the target nucleus. Over the past few years, Fermilab's MINERvA experiment has produced several studies of quasielastic scattering using the low-energy NuMI beam, which peaks in the $3 \mathrm{GeV}$ region. I will present a selection of results for both neutrino and antineutrino scattering on scintillator, including the world's first double-differential cross sections in this energy range, explaining what these can tell us about nuclear effects. In addition I will show a new result which demonstrates how the observed quasi-elastic cross section changes when scattering from different nuclei.

The 19th International Workshop on Neutrinos from Accelerators-NUFACT2017

25-30 September, 2017

Uppsala University, Uppsala, Sweden

${ }^{*}$ Speaker.

${ }^{\dagger}$ For the MINERvA collaboration. 


\section{The MINERvA experiment}

MINERvA is situated in Fermilab's NuMI beam [1]. In its low-energy configuration, between 2010 and 2012, we collected approximately $3 \times 10^{20}$ protons on target (POT) of neutrino-mode and $10^{20}$ POT of antineutrino data, with a peak energy around $3 \mathrm{GeV}$, which was used to produce the analyses described in these proceedings.

MINERvA's detector, described in detail in [2], has a fully-instrumented tracker consisting of hexagonal planes of plastic $(\mathrm{CH})$ scintillator perpendicular to the beam. Each plane consists of 127 triangular scintillator strips, in an alternating pattern of three orientations, allowing threedimensional reconstruction. Lead and steel calorimeters downstream and on the sides of the detector let us contain and determine the energy of hadrons, electrons and gammas. The MINOS near detector, downstream, acts as a muon calorimeter. In the upstream 'nuclear target' region of the detector, passive materials between the active planes let us study the cross section's $A$-dependence.

\section{The state of quasi-elastics at MINERvA}

The charged-current quasi-elastic (CCQE) interaction is a golden channel in oscillation experiments, due to its characteristic and recognisable signature. On a free nucleon, the interaction takes the form $v_{\mu}+n \rightarrow p+\mu^{-}$or $\bar{v}_{\mu}+p \rightarrow n+\mu^{+}$. Figure 1 shows characteristic neutrino (1a) and antineutrino (1b) event signatures in the MINERvA event viewer, Arachne [3].

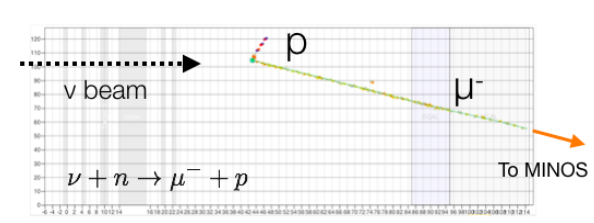

(a) Neutrino mode

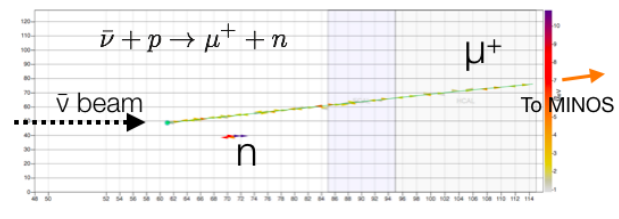

(b) Antineutrino mode

Figure 1: Quasi-elastic scattering in MINERvA. (Neutron not always visible in $\bar{v}$ mode.)

In 2013, MINERvA published CCQE cross sections vs. the squared four-momentum transfer $Q^{2}$ for both muon neutrinos [4] and antineutrinos. [5] These results showed poor agreement with our simulation, GENIE 2.6.2 [6], which simulated the effect of the nucleus with a relativistic Fermi gas model [7] and INTRANUKE-hA cascade model [8] for final state interactions. This hinted at the presence of additional effects. Subsequent analyses, described in this paper, have attempted to investigate the possible mechanisms for this discrepancy.

\section{Evaluating multi-nucleon effects}

\subsection{Multi-nucleon correlations}

The basic Fermi Gas [7] models the Fermi momentum distribution of nucleons within the nucleus, and Pauli blocking [9], but neglects the effects of correlations between two or more of nucleons. Short range correlations are already modelled in GENIE by a Bodek-Ritchie momentum 
tail [10]. Medium-range correlations due to meson-exchange currents (MEC) [11] and long-range RPA effects [12] are not included by default in our current version of GENIE (2.8.4), but are available from newer versions.

We cannot measure neutrino energy directly so, for the CCQE channel, we reconstruct it from muon kinematics. However, the formula [13] assumes independent nucleons. Scattering from correlated pairs causes incorrect energy reconstruction. We refer to this as two-particle-two-hole (2p2h) scattering. Additionally, when scattering from a correlated pair, the partner nucleon may be ejected, leaving a final state with a second, unexpected nucleon, and making it harder to identify quasi-elastic events.

Electron-scattering experiments found that, approximately $20 \%$ of the time, electrons scattered from correlated pairs of nucleons instead of single nucleons. $90 \%$ of these pairs consisted of a proton and a neutron [14]. In electron-nucleus scattering experiments, these events correspond to an excess in the cross section in the dip region between the quasi-elastic and $\Delta$ resonance peaks, when plotted versus energy transfer and total electron momentum.

\subsection{Strength of MEC and 2p2h in MINERvA}

To evaluate the strength of multi-nucleon effects, we seek to emulate the phase space used in electron-scattering experiments. By looking at simulation (GENIE 2.8.4 tuned to match [15]) in the total energy transfer $q_{0}$ and three-momentum transfer $q_{3}$, we can distinguish the regions in which different interaction types take place, and isolate where the cross section is enhanced by 2p2h effects, and suppressed by RPA (Figure 2) [16]. The multi-nucleon effects are modelled using the IFIC Valencia model of 2p2h [17][18] and Nieves model of RPA [19].

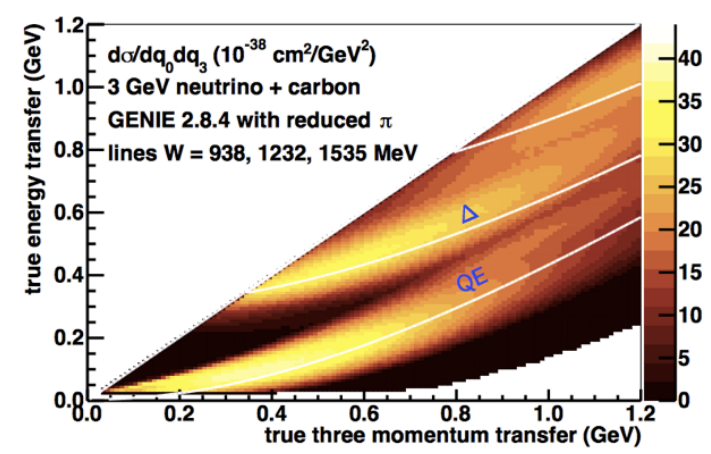

(a) CCQE and $\Delta$ regimes in simulation

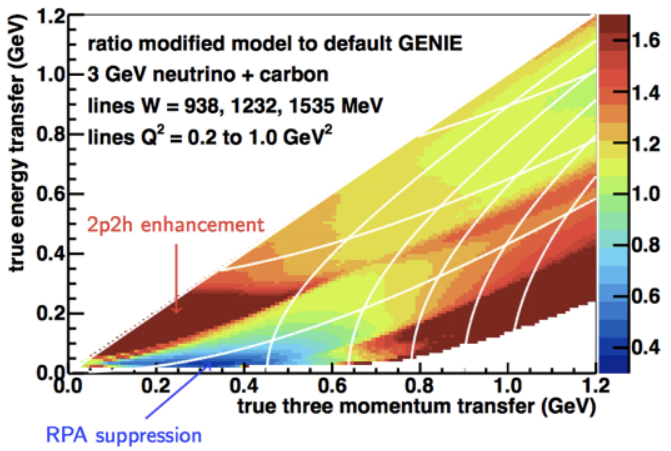

(b) Effect of MEC and RPA on the cross section

Figure 2: Simulation of the inclusive neutrino-nucleus scattering cross section in MINERvA, in the $q_{0}-q_{3}$ phase space that distinguishes between interaction types.

To compare with MINERvA data, we use calorimetrically measured energy to reconstruct $q_{0}$ and $q_{3}$. However, as MINERvA is poor at seeing neutrons, the best we can do is sum the available reconstructed hadronic energy $E_{\text {avail }}$, corresponding to $q_{0}$ minus neutron energy. In neutrino mode, we expect this to be a small effect, but it renders this method impractical for antineutrino mode. While adding RPA improved the fit at low energies, there was still a deficit in the dip region when compared to GENIE. Proton multiplicity in this region (identified by Bragg peak $>20 \mathrm{MeV}$ ) was 
also higher than predicted [20]. Weighting up the 2p2h contribution with a 2-d Gaussian multiplier in $q_{0}-q_{3}$ space improved the fit, due to additional events from initial $n p$ pairs (yielding a $p p$ final state). While the total increase was around $60 \%$, it was concentrated in the dip region between the quasi-elastic and $\Delta$ resonance peaks (see Figure 3). Although an equivalent analysis could not be performed in $\bar{v}$ mode, adding RPA and 2p2h scaled to the $v$ mode fit also improved $\bar{v}$ agreement.

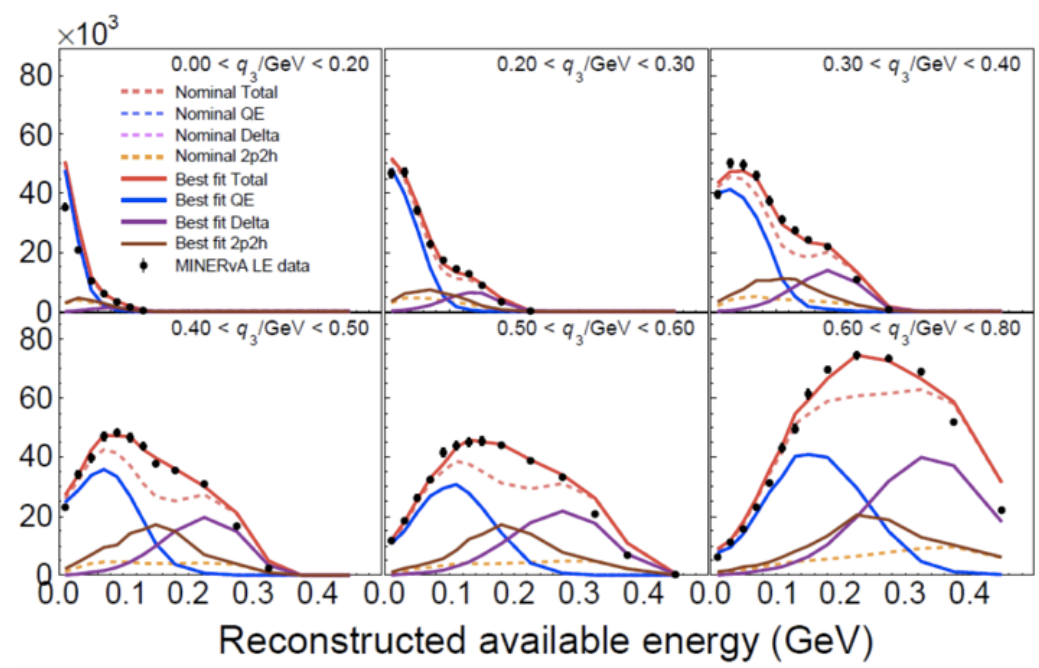

Figure 3: Best fit to data, with $2 \mathrm{p} 2 \mathrm{~h}$ contribution scaled by a Gaussian multiplier in $q_{0}-q_{3}$ space

\section{Double-differential cross sections}

In response to the study mentioned in Section 3.2, the nominal GENIE 2.8.4 simulation was modified to include the IFIC $2 \mathrm{p} 2 \mathrm{~h}$ model, tuned to MINERvA data [20] and RPA [19]. Furthermore, it was found necessary to tune down the non-resonant pion production [15]. Using the latest NuMI flux [21], we produced double-differential cross sections for both neutrinos and antineutrinos. To ensure good phase space coverage, and because the variables were directly measurable, it was decided to produce cross sections as a function of muon longitudinal and transverse momentum $\left(p_{\|}\right.$and $p_{T}$ ). In most of our phase space, these loosely correspond to $E_{V}$ and $Q^{2}$ respectively.

In contrast to our 2013 analyses, and for consistency with other experiments, we used a "quasielastic-like" signal definition, corresponding to events with a muon of appropriate charge and no pions in the final state $(C C O \pi)$. In addition, for the antineutrino analysis, we defined our signal as having a true final state that included no protons with kinetic energy above $120 \mathrm{MeV}$ : these events frequently produce a second track and resemble background far more than they resemble a $\bar{v}$ CCQE signature. As our muon charge reconstruction requires the muon to be tracked downstream into the MINOS near detector, limiting angular acceptance, a maximum muon angle of $20^{\circ}$ was required.

For the antineutrino analysis, events were selected with a reconstructed $\mu^{+}$track and no other reconstructed tracks. A $Q^{2}$-dependent cut was also made on recoil energy - untracked energy more than $10 \mathrm{~cm}$ from the reconstructed muon vertex [22]. For the neutrino analysis, track $d E / d x$ was used to distinguish protons from pions. Tracks with more than one isolated energy deposit, and tracks with the delayed energy consistent with a Michel electron were vetoed, as were events with more than $500 \mathrm{MeV}$ of recoil energy. 
To reduce model dependence, data-driven methods were used to subtract backgrounds. In antineutrino mode, the shapes of the signal and background distributions were fitted with ROOT's [23] TFractionFitter algorithm, giving a background scale corresponding to the best fit to data shape. In neutrino mode, three sidebands were used to estimate background: events with Michel electrons, with more than one isolated energy deposit, and with both.

Again, for both neutrinos (Figure 4) and antineutrinos (Figure 5), the MINERvA-tuned GENIE gave a significant improvement in fit, indicating that the results of the inclusive measurement apply well to quasi-elastic distributions (Figure 4).

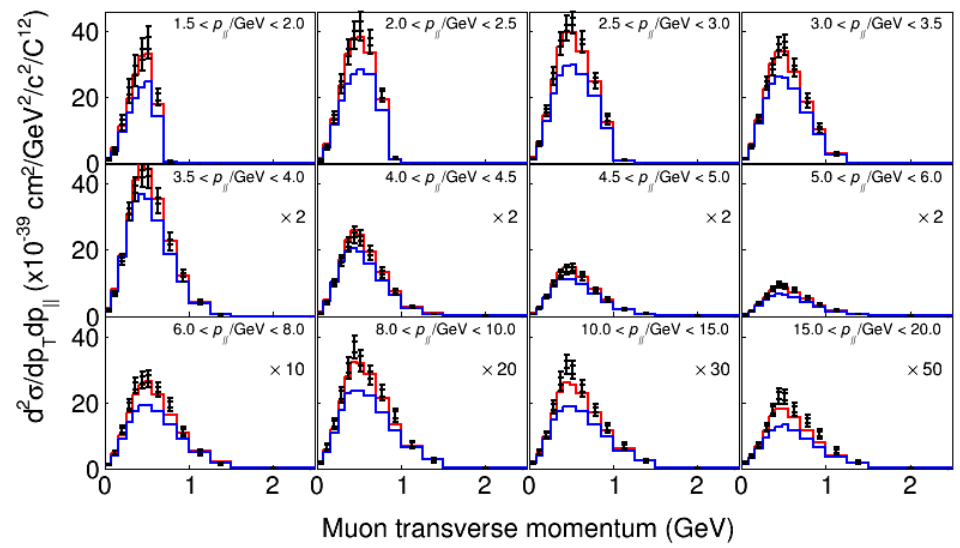

Figure 4: Comparing $v$-mode cross section with default (blue) and MINERvA-tuned (red) GENIE
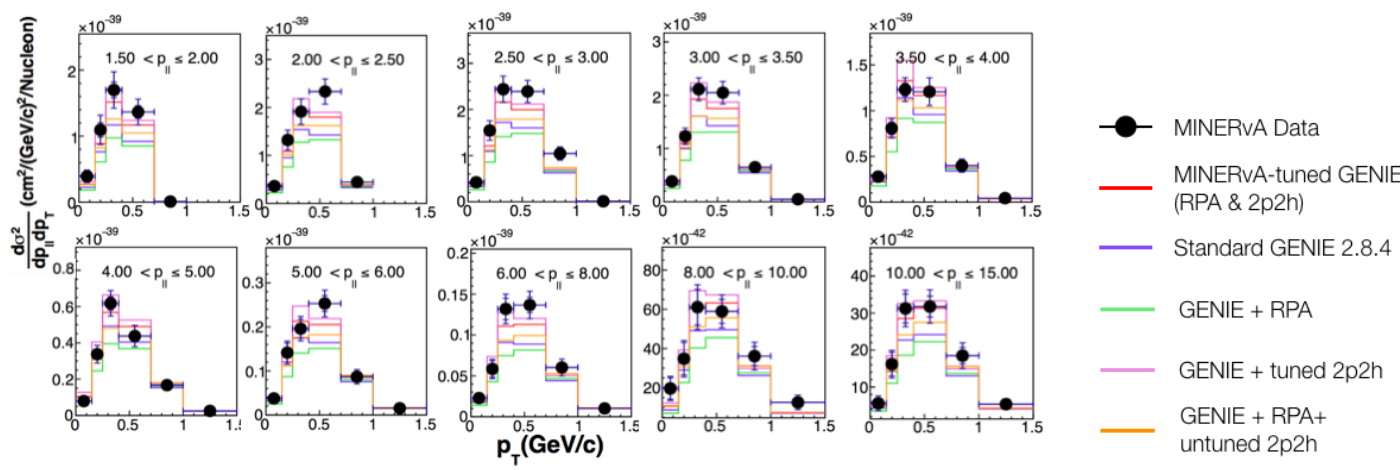

Figure 5: Antineutrino cross section compared with various models.

\section{Quasi-elastic scattering on nuclear targets}

As described in [2], the upstream section of MINERvA includes several passive nuclear targets, arranged with combinations of iron, lead and carbon (graphite) in different geometrical locations to reduce systematic uncertainty. A quasi-elastic-like cross section was measured for each material. The signal was an event with at least one proton above $450 \mathrm{MeV} / \mathrm{c}$, one negative muon, and no pions, from the appropriate material. We require a muon-like track (no MINOS-match requirement) and proton-like track stopping in the detector, with an extrapolated vertex in the selected 
material. Pions are removed with a $\mathrm{dE} / \mathrm{dx}$ check and Michel electron veto, and a recoil energy cut is applied. Backgrounds of events in the scintillator surrounding the targets, and of events with pions, are removed by looking at upstream and downstream sidebands, and by fitting in recoil energy.

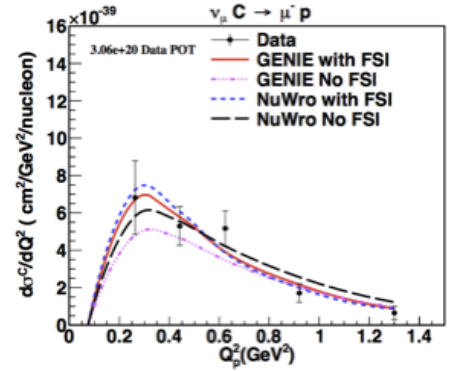

(a) $\operatorname{Carbon}(\mathrm{A} \approx 12)$

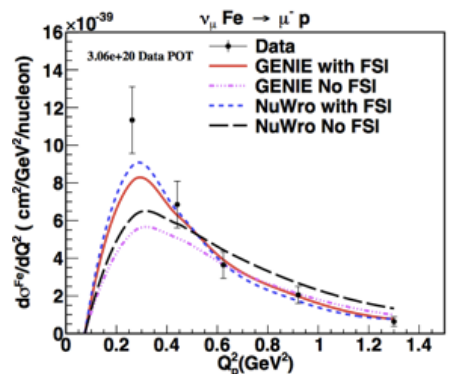

(b) $\operatorname{Iron}(\mathrm{A} \approx 56)$

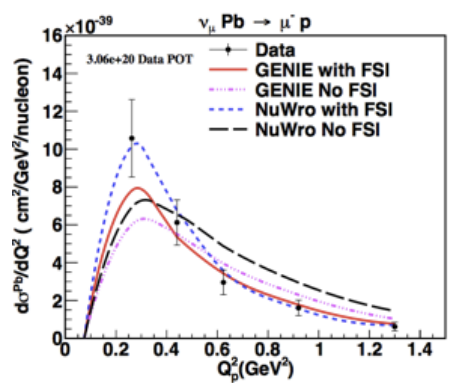

(c) Lead $(\mathrm{A} \approx 207)$

Figure 6: Nuclear target cross sections compared with GENIE and NuWro, with and without FSI.

Figure 6 (from [24]) shows cross sections for the three materials, compared with GENIE and NuWro's [25] simulations (both including similar 2p2h and RPA effects) with and without finalstate interaction models. In each case, the best agreement is with NuWro with FSI, which incorporates an $A$-dependent pion absorption FSI model not included in GENIE. However, plotting the coplanarity angle (angle between the $v-\mu$ and $v-p$ planes, which should be $180^{\circ}$ for a free nucleon) shows more spread than should be expected from modelled initial and final-state interaction smearing. This increases with heavier materials, indicating our FSI models are still incomplete.

\section{Conclusion}

MINERvA's tuned simulations now do a good job of reproducing data for both $v$ and $\bar{v}$ quasielastic cross sections - but we do not yet have a theoretical motivation for our tuning. The next step is to find an explanation for why this scaling of the $2 \mathrm{p} 2 \mathrm{~h}$ effect produces a good fit.

\section{References}

[1] P. Adamson et al., The NuMI neutrino beam, Nucl. Inst. and Meth. A 806 (2016) 279 - 306 , [1507.06690].

[2] MINERvA collaboration, L. Aliaga et al., Design, Calibration, and Performance of the MINERvA Detector, Nucl. Inst. and Meth. A 743 (2014) 130-159, [1305. 5199].

[3] MINERvA collaboration, N. Tagg et al., Arachne - A web-based event viewer for MINERvA, Nucl. Instrum. Meth. 676 (2012) 44-49, [1111. 5315].

[4] MINERvA collaboration, G. A. Fiorentini et al., Measurement of Muon Neutrino Quasielastic Scattering on a Hydrocarbon Target at $E_{v} \sim 3.5 \mathrm{GeV}$, Physical Review Letters 111 (July, 2013) 022502, [1305.2243].

[5] MINERvA collaboration, L. Fields et al., Measurement of Muon Antineutrino Quasielastic Scattering on a Hydrocarbon Target at $E_{V} \sim 3.5$ GeV, Physical Review Letters 111 (July, 2013) 022501, [1305.2234]. 
[6] C. Andreopoulos et al., The GENIE neutrino Monte Carlo generator, Nucl. Inst. and Meth. A 614 (2010) $87-104$, [0905.2517].

[7] R. A. Smith and E. J. Moniz, Neutrino reactions on nuclear targets, Nuclear Physics B 43 (1972) 605 -622 .

[8] S. Dytman, INTRANUKE-hA, in AIP Conf Proc, vol. 896, pp. 178-184, 2007.

[9] L. Liu, Pauli blocking and final-state interaction in electron-nucleus quasielastic scattering, Phys. Rev. C 79 (Jan, 2009) 014605, [0901.3561].

[10] A. Bodek and J. L. Ritchie, Fermi-motion effects in deep-inelastic lepton scattering from nuclear targets, Phys. Rev. D 23 (Mar, 1981) 1070-1091.

[11] J. W. Van Orden and T. W. Donnelly, Mesonic processes in deep-inelastic electron scattering from nuclei, Annals of Physics 131 (1981) 451 - 493.

[12] J. Morfín, J. Nieves and J. Sobczyk, Recent developments in neutrino/antineutrino-nucleus interactions, Advances in High Energy Physics 2012 (2012) 934597, [1209. 658 6].

[13] C. Llewellyn Smith, Neutrino reactions at accelerator energies, Physics Reports 3 (1972) 261-379.

[14] R. Subedi et al., Probing cold dense nuclear matter, Science 320 (2008) 1476-1478.

[15] C. Wilkinson, P. Rodrigues, S. Cartwright, L. Thompson and K. McFarland, Reanalysis of bubble chamber measurements of muon-neutrino induced single pion production, Phys. Rev. D 90 (Dec, 2014) 112017, [1411.4482].

[16] R. Gran, J. Nieves, F. Sanchez and M. J. V. Vacas, Neutrino-nucleus quasi-elastic and $2 p 2 h$ interactions up to 10 GeV, Phys. Rev. D 88 (Dec, 2013) 113007, [1307.8105].

[17] P. Coloma, P. Huber, C.-M. Jen and C. Mariani, Neutrino-nucleus interaction models and their impact on oscillation analyses, Phys. Rev. D 89 (Apr, 2014) 073015, [1311. 4506 ].

[18] J. Schwehr, D. Cherdack and R. Gran, GENIE implementation of IFIC Valencia model for QE-like 2p2h neutrino-nucleus cross section, 1601.02038.

[19] J. Nieves, J. E. Amaro and M. Valverde, Inclusive quasielastic charged-current neutrino-nucleus reactions, Phys. Rev. C 70 (Nov, 2004) 055503, [0 408005 ].

[20] MINERvA collaboration, P. A. Rodrigues et al., Identification of nuclear effects in neutrino-carbon interactions at low three-momentum transfer, Phys. Rev. Lett. 116 (Feb, 2016) 071802, [1511.05944].

[21] MINERvA collaboration, L. Aliaga et al., Neutrino flux predictions for the NuMI beam, Phys. Rev. D 94 (Nov, 2016) 092005, [1607.00704].

[22] C. E. Patrick, Measurement of the Antineutrino Double-Differential Charged-Current Quasi-Elastic Scattering Cross Section at MINERvA. Springer International, 2017, 10.1007/978-3-319-69087-2.

[23] R. Brun and F. Rademakers, ROOT: An object oriented data analysis framework, Nucl. Instrum. Meth. A389 (1997) 81-86.

[24] MINERvA collaboration, M. Betancourt et al., Direct Measurement of Nuclear Dependence of Charged Current Quasielasticlike Neutrino Interactions Using MINERvA, Phys. Rev. Lett. 119 (Aug, 2017) 082001, [1705.03791].

[25] T. Golan, J. T. Sobczyk and J. Zmuda, NuWro: the Wroclaw Monte Carlo Generator of Neutrino Interactions, Nucl. Phys. Proc. Suppl. 229-232 (2012) 499. 